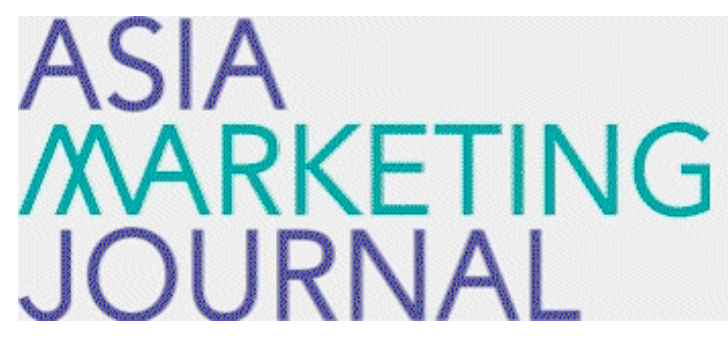

ASIA MARKETING JOURNAL

Volume 20 | Issue 2

Article 1

7-30-2018

\title{
A Future Contraction Effect in Intertemporal Choice for Durable Goods
}

Byung Kyu Kim

Follow this and additional works at: https://amj.kma.re.kr/journal

Part of the Marketing Commons

\section{Recommended Citation}

Kim, Byung Kyu (2018) "A Future Contraction Effect in Intertemporal Choice for Durable Goods," Asia Marketing Journal: Vol. 20 : Iss. 2 , Article 1.

Available at: https://doi.org/10.15830/amj.2018.20.2.29

This Article is brought to you for free and open access by Asia Marketing Journal. It has been accepted for inclusion in Asia Marketing Journal by an authorized editor of Asia Marketing Journal. 


\title{
A Future Contraction Effect in Intertemporal Choice for Durable Goods
}

\author{
Byung Kyu Kim*
}

\begin{abstract}
Recent research reported that perception of future time is non-linearly scaled. That is, as objective time gets longer, subjective perception of the objective time does not grow proportionally. The non-linear time perception implies that the same future time feels shorter when it starts in the future than when it starts immediately. The authors call this as a future contraction effect. The current research tests two important implications of the effect regarding consumers' intertemporal preference for durable goods. First, consumers who contract future more will be more impatient for durable goods compared to those who contract less because the former would feel to use the same durable goods longer when it is purchased immediately. Second, consumers' impatience will be alleviated when their tendency to contract future is reduced. The authors find support for these predictions through two studies. Taken together, the current research demonstrates a property of time perception that has important ramifications for understanding consumers' intertemporal preference for durable goods.
\end{abstract}

Key words: time perception, usage duration, durable goods, preference, intertemporal choice

Studies in psychophysics have demonstrated that internal representation of the magnitude of external stimuli is non-linearly scaled (Stevens 1957). This property of non-linear scaling in human sensation and perception has been demonstrated for various types of stimuli, such as brightness or loudness. Recently, marketing researchers have proposed that perception of future time is also non-linearly scaled with a power exponent less than one (Ebert and Prelec 2007; Killeen 2009; Kim and Zauberman 2009; Loewenstein and Prelec 1992; Zauberman et al. 2009). That is, as objective time gets longer, subjective perception of the objective time does not grow proportionally. Such non-linear scaling in future time perception implies that the same future interval may be perceived to be shorter as its start point becomes more

\footnotetext{
* Associate Professor of Marketing at Yonsei University School of Business (kyukim@yonsei.ac.kr)
} 
distal from the present. For instance, the same one-month interval may feel shorter when it starts in one year than when it starts today. In this research, we call this as a future contraction effect.

The future contraction effect is an interesting phenomenon by itself. But what does it imply for understanding consumer behavior? The current research aims to address this simple but important question for marketing research and practice. In particular, based on the future contraction effect, we draw two novel hypotheses regarding consumers' intertemporal preference for durable goods. First, we predict that those consumers who contract future time more would be more impatient for durable goods compared to those who contract it less. If consumers perceive the same future interval to be shorter when it starts in the future, they would expect to use the same durable good for a shorter period when it is purchased in the future than when it is purchased immediately. Hence, for those who contract future more, the same durable available in the future would look less attractive compared to those who contract future less, and, as a result, the former would become more impatient for the durable good. Second, we predict that consumers' impatience can be alleviated simply by changing how much they contract future time. Prior research has demonstrated that time perception is an important determinant of consumers' intertemporal preference (Kim and Zauberman 2009; Zauberman et al. 2009). Building on the relationship between time perception and intertemporal preference, we expect to influence consumers' impatience for a durable good simply by changing how much they contract their future. We find support for these hypotheses through two studies. The first study supports our hypotheses by analyzing correlational data and the second study uses an experimental method. In the following sections, we discuss the theoretical background of our predictions more in detail.

\section{Theoretical Development}

\subsection{Future Contraction Effect}

Many decisions consumers make involve judgment of future time. When there are sales promotions in the future, consumers consider whether they want to wait for the promotions or just make purchases on that day. In making such decisions, their decisions to purchase now versus later should be influenced by how long they subjectively feel the waiting time to be. Prior research has directly measured consumers' perception of future time and found that perceived time is not a linear function of actual time but non-linearly scaled with a power exponent less than one (Kim and Zauberman 2009; Zauberman et al. 2009). For example, Zauberman et al. (2009) showed that participants 
perceived a 12-month interval not as 4 times but only as 1.24 times longer than a three-month interval. Figure 1 illustrates the non-linear scaling in future time perception.

Such non-linear scaling in future time perception implies that the same interval may be perceived to be shorter when it starts in the future than when it starts in the present. For instance, in Figure 1, a subjectively perceived three-month duration starting after nine months (' $b$ ') is shorter than a subjectively perceived three-month duration starting immediately ('a’). Although the possibility of such future contraction has been proposed by multiple researchers (Killeen 2009; Kim and Zauberman 2009; Loewenstein and Prelec 1992; Zauberman et al. 2009), direct empirical tests of the future contraction effect are still very scant. Hence, the first goal of the current investigation is to demonstrate the existence of the future contraction effect. For this purpose, we hypothesize that,

H1: Participants will perceive the same future interval to be shorter when it starts in the future rather than from today.

What does the future contraction effect imply for our understanding of consumer behavior? Prior research has documented one phenomenon that the effect can explain, hyperbolic discounting. In many economic model of consumer behavior, researchers often assume that one's discount rate does not change depending on when consumption occurs. For example, regardless of whether a consumer delays $\$ 100$ from today to tomorrow, or she delays the same amount

〈Figure 1〉 Non-linear scaling in time perception

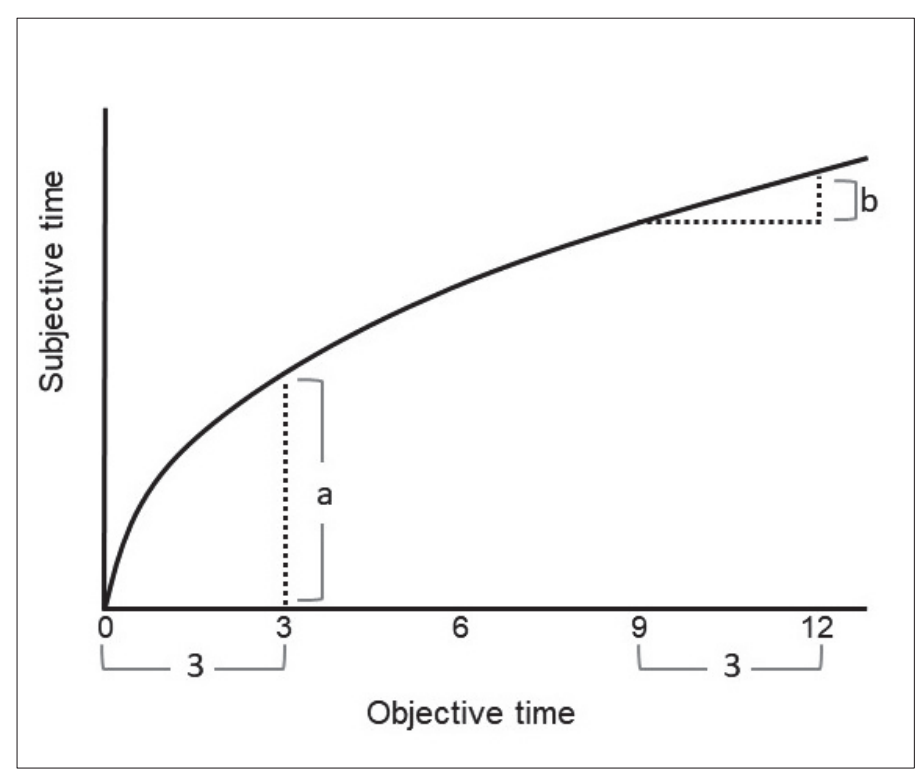


money from 10 days later to 11 days later, her discount rates for one-day delay should be the same in two occasions. But numerous empirical tests proved that it is not the case (e.g., Thaler 1981). Specifically, when consumers delay their consumption from today to tomorrow, they are much more reluctant to delay their consumption compared to when they delay it from 10 days later to 11 days later. When one's discount rates were elicited, discount rates are highest when giving up immediate consumptions and they decrease as consumption is pushed to the future. This phenomenon is often denoted as hyperbolic discounting. Researchers proposed that hyperbolic discounting may be the outcome of non-linear scaling (Killeen 2009; Kim and Zauberman 2009; Zauberman et al. 2009). That is, because consumers perceive the same length of delay to be longer when it starts from today than when it starts in the future, they are more impatient when they have to give up immediate consumptions than when they are giving up future consumptions.

Although hyperbolic discounting is a very important phenomenon, this has been the only one that researchers have attempted to explain using non-linear scaling in future time perception. Hence, in the current research, we aim to test novel predictions that we draw from the future contraction effect. In particular, we aim to show an important implication of the effect regarding consumers' intertemporal preference for durable goods.

\subsection{Intertemporal Choice for Durable Goods}

Durable goods such as washing machines and televisions, or even service goods such as gym memberships, provide a stream of benefits over the duration of their usage. Consumers often face situations in which they make decisions to purchase a durable good either immediately or after a delay. They may delay their purchase because of, for instance, promotions scheduled in the future or the condition of the good they currently own. Such decisions that involve choices between immediate purchases and future purchases are referred as intertemporal choices.

We propose that the future contraction effect serves as a unique determinant of intertemporal preference for durable goods. Usage duration for durables is temporal information. Hence, if the future contraction effect exists for the subjective perception of future time in general, it should emerge for the perception of usage duration as well. For instance, consumers may subjectively feel that they are going to use the same durable for a shorter period of time if they purchase it in the future than if they purchase it immediately. That is, usage duration of a durable good to be purchased in the future will be contracted compared to the same length of usage duration for a durable good purchased immediately. Based on this reasoning, we draw the following two hypotheses.

First, we hypothesize that heterogeneity among 
consumers in contracting future time would predict their intertemporal preferences for durable goods. Although we posit that a future contraction effect is a general phenomenon, there may be an individual difference in how much consumers contract future time. Some consumers would perceive usage duration in the future to be very short while other consumers would perceive it relatively longer. Then, those who contract future time more are more likely to opt for immediate purchases because usage duration of durables to be purchased in the future seems shorter and, therefore, such durables look less attractive as compared to those who contract future time less. Hence, we predict that those consumers who contract future time more are more likely to choose immediate purchases of a durable good over a delayed one compared to those who contract it less. In other words, those who contract future more will be more impatient for a durable good compared to those who contract it less. To test this possibility, we hypothesize that,

H2: Participants who contract future time more will be more impatient for a durable good compared to those who contract future time less.

Second, we hypothesize that consumers' impatience for durable goods can be alleviated by changing how much they contract future time. Consumers' impatience is known be associated with various problematic behaviors such as overspending, obesity, substance abuse, addiction, or even low credit scores (Chabris et al. 2008; Bickel and Marsch 2001; Kirby and Petry 2004; Meier and Sprenger 2012). Hence, it is very important to find ways to reduce consumers' impatience. Our premise that consumers' intertemporal preference for durable goods is associated with their tendency to contract future time implies that if we change how much they contract future, we may be able to influence their impatience as well. Suppose that a consumer is deciding whether to purchase a durable today or later in the future. If she contracts future usage duration very heavily as compared to present usage duration, the durable good to be purchased in the future would look very unattractive because she feels to use it only for a short period of time. Hence, the consumer will show impatience for the durable good. However, if we make the consumer contract future time to a less degree, the future alternative would look more attractive and her impatience will be attenuated. To test this, we hypothesize the following.

H3: When participants' tendency to contract future time is reduced, their impatience for immediately available durables over durables available in the future will be attenuated.

To summarize, the current research aims to 
demonstrate important implications of the future contraction effect. We test our hypotheses through two studies. In Study 1, we test whether participants' tendency to contract future is indeed associated with their intertemporal preference for a durable good. In Study 2, we experimentally manipulate participants' tendency to contract future and examine whether it influences their intertemporal preference for a durable good.

\section{Study 1}

Study 1 aims to test hypotheses 1 and 2. For this purpose, we measured participants' future time perception and their intertemporal preference for a durable good (a smartphone), and examined whether they are associated.

\subsection{Method}

Participants were eighty-six adults from an online panel $\left(\mathrm{M}_{\text {age }}=32.41, \mathrm{SD}_{\text {age }}=12.38 ; 34\right.$ females). This study consisted of two parts: a time perception task and an intertemporal choice task for a smartphone. In the time perception task, we measured participants' perception of two future intervals: one starting from today and the other starting in 12 months. Specifically, we asked them to think about a 24-month duration starting in the present (e.g., today) and to indicate their subjective feeling of duration. Participants indicated their feeling using a computerized string. On the next page, we asked them to think about another 24-month duration but starting in 12 months.

In the intertemporal choice task, we asked participants to imagine that they were considering buying a smartphone and found a model that they liked very much at a local store. We further asked them to imagine they were going to use the smartphone at least 24 months once they purchase it and were considering whether to buy this smartphone today or in 12 months. We told them if they buy it on that day, they will pay $\$ 250$, but if they wait for 12 months, they will pay $\$ 250$ but will receive $\$ 100$ instant cash rebate. Then, they indicated their relative preference between the two intertemporal outcomes. Specifically, they indicated which one they preferred between the immediate option and the delayed option on an 11-point scale $(1=$ strongly prefer to buy it today, $5=$ indifferent (or equally prefer both options), $9=$ strongly prefer to buy it in 12 months).

\subsection{Results and Discussion}

First, we tested the future contraction effect (hypothesis 1). Participants' responses for time perception using the computerized string were recorded in millimeters. Consistent with the practices in psychophysics, these responses were log transformed. Results showed that participants judged the same 24-month duration to be 
shorter when it starts in 12 months $(\mathrm{M}=$ $287.64 \mathrm{~mm}, \mathrm{SD}=172.00)$ than in the present $(\mathrm{M}=266.38 \mathrm{~mm}, \mathrm{SD}=226.60 ; \mathrm{t}(85)=2.09$, $p<.05)$. This result confirms the future contraction effect that the same interval feels shorter when it starts in the future than from the present.

Next, we tested whether the degree of future contraction is associated with their intertemporal preference for a smartphone. As an indicator of contraction, we calculated the difference between participants' two responses. Intertemporal preference was participants' responses on the 11 -point scale $(\mathrm{M}=4.84, \mathrm{SD}=3.10)$. We found that the degree of contraction for each participant was significantly correlated with their intertemporal preference for a smartphone $(\mathrm{r}=$ $-.22, p=.04)$. Specifically, as participants contract future time more, they preferred to buy the smartphone on that day more strongly. This result confirms hypothesis 2 and demonstrates that participants' degree of future contraction is related to their intertemporal preference for a durable good.

\section{Study 2}

Study 2 aims to test hypothesis 1 and 3. In particular, through an experimental method (e.g., providing a visual cue depicting the length of future time), we aim to reduce participants' degree of future contraction. We predict that such treatment will alleviate their impatience for an immediately available durable good.

\subsection{Method}

One hundred and eighty adults from an online panel completed the study $\left(\mathrm{M}_{\text {age }}=30.26\right.$, $\mathrm{SD}_{\text {age }}=8.94 ; 76$ females, 1 unknown). They were randomly assigned to either visual-cue or a no-visual-cue conditions.

We measured participants' intertemporal preference for a durable using a titration task where participants made a series of choices between an immediate option and a delayed option (Hardisty and Weber 2009). Specifically, participants imagined that they recently won a raffle and will receive a \$1000-value washing machine on that day. Then, we asked them whether they could wait to receive the washing machine for 12 months. If they were willing to wait for 12 months to receive the washing machine, they would receive a cash prize to compensate for the delay. On the following pages, we presented them with a series of choices, one choice on each page. First, we asked them which one they preferred between 'A. Receive the \$1000-value washing machine today' or 'B. Wait for 12 months and receive the washing machine $+\$ 20^{\prime}$. If they chose $A$, then we directed them to the next page where they were presented with another choice task, in which the value of the cash prize for the option 
$\mathrm{B}$ was increased by $\$ 20$ (e.g., \$40). If they chose $\mathrm{B}$, then this choice task was terminated. Whenever participants chose the immediate option, the value of the cash prize with the future option was increased by $\$ 20$. The maximum value of the cash prize was set to be $\$ 800$.

As our key experimental manipulation, we presented participants in the visual-cue condition with a visual image of usage durations when they made their choice between an immediate and a delayed alternative. In a purpose of reducing the future contraction effect, usage durations are graphically depicted in an equal length regardless of whether it starts on that day or in 12 months (see Figure 2). Participants in the no-visual-cue condition made intertemporal choices with no visual cues of durations.

After participants completed their intertemporal choice tasks, we measured their subjective perception of usage durations. Specifically, we asked participants to imagine that they received a washing machine on that day and that they were going to use it for 10 years. Then, they indicated their subjective feeling of the 10year usage duration. In doing so, we provided them with a horizontal bar scale where a left end point was labeled as 'very short' and the right end point was labeled as 'very long'. This scale coded participants' responses between 0 and 100. Next, we asked them to imagine that they were going to receive a washing machine not on that day but in 12 months, and that they were going to use it for 10 years after they received it in 12 months. Then, they indicated their feeling of the 10-year duration using a similar horizontal bar scale.

\subsection{Results and Discussion}

\subsubsection{Future contraction effect}

Participants' responses for the subjective perception of the 10-year duration starting on that day and starting in 12 months were log transformed for analyses. We first examined whether there is a future contraction effect for participants in the no-visual-cue condition. Demonstrating the future contraction effect, we found that participants in this condition perceived the same 12-month usage duration to be shorter when it starts in 12 months than immediately on that day $\left(\mathrm{M}_{\text {today }}=4.38, \mathrm{SD}=\right.$ .25 vs. $\mathrm{M}_{12 \text { months }}=4.22, \mathrm{SD}=.57 ; \mathrm{F}(1,178)=$ 10.51, $p<.001)$. Participants in the visual-cue

〈Figure 2〉 Visual cue presented in Study 2

\section{A. Washing Machine}

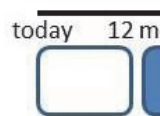

B. Washing Machine + Cash Prize 
condition, however, did not show evidence for the future contraction effect: their perception of the 12-month usage duration were not different regardless of when it starts $\left(\mathrm{M}_{\text {today }}=\right.$ 4.34, $\mathrm{SD}=.29$ vs. $\mathrm{M}_{12 \text { months }}=4.32, \mathrm{SD}=.35$; $\mathrm{F}(1,178)=.08, p=.77)$. These results confirm the existence of the future contraction effect in the perception of usage duration of a durable good. They also show that our experimental manipulation was successful.

\subsubsection{Intertemporal preference for a washing machine}

Participants' intertemporal preference was determined when they switched their choice from an immediate option to a delayed option. For instance, if they chose the immediate option (e.g., \$1000-value washing machine today) over a delayed option with $\$ 40$ delay premium (e.g., \$1000-value washing machine + \$40 in 12 months), but switched their choice when the delay premium becomes $\$ 60$, then intertemporal preference for this participant was calculated as the mid-point of two delay premiums (e.g., \$50). If they did not choose the delayed option until the last choice pair, where the delay premium is $\$ 800$, their indifference price was set to be $\$ 800$. Consistent with past studies, the corresponding delay premium values were log transformed for further analyses. We then examined whether participants' intertemporal preference was different between the two conditions. Confirming our prediction, results showed that participants in the no visual-cue condition placed a greater value for an immediatelyavailable washing machine than those who in the visual-cue condition $\left(\mathrm{M}_{\text {no-visual-cue }}=5.06\right.$, $\mathrm{SD}=1.02$ vs. $\mathrm{M}_{\text {visual-cue }}=4.62, \mathrm{SD}=1.27$; $t(178)=2.58, p=.01)$. That is, when participants' future contraction was experimentally reduced, their impatience level for an immediately available durable good decreased as well. These results confirm hypothesis 3 .

\section{General Discussion}

In the current research, we demonstrated a future contraction effect and its implication for consumers' intertemporal preference for durable goods. Specifically, we demonstrated the future contraction effect in the perception of future time (Study 1) and in the perception of usage duration (Study 2). Then, we showed that one's degree to contract future time is associated with their intertemporal preference for a durable (Study 1), and one's impatience can be alleviated by reducing how much she contracts future time (Study 2). Taken together, our findings show a property of time perception that has important ramifications for consumers' intertemporal preference for durables. Next, we discuss broader implications of our findings and future research. As we discussed, many researchers have 
proposed the possibility of future contraction (Ebert and Prelec 2007; Killeen 2009; Kim and Zauberman 2009; Loewenstein and Prelec 1992; Zauberman et al. 2009). To the best of our knowledge, no past studies have directed demonstrated a future contraction effect. Hence, the current research is the first empirical investigation that shows the future contraction effect. The current research also contributes to the intertemporal choice literature by demonstrating a unique factor (i.e., the future contraction effect) that contributes to intertemporal preference for durable goods. Intertemporal choice literature has identified various factors influencing impatience in intertemporal choices such as emotional responses to immediate consumptions (Lowenstein 1996), perception of waiting time (Bilgin and LeBoeuf 2010; Ebert and Prelec 2007; Kim and Zauberman 2009; Zauberman et al. 2009), or perceived connectedness between present and future selves (Bartels and Rips 2010; Bartels and Urminsky 2011). Note, however, that most of these studies have been conducted in the context of intertemporal decisions regarding monetary outcomes. But consumers make intertemporal decisions not only for money but also for various durable goods such as electronics, automobiles, and even houses. Hence, investigating factors uniquely associated with impatience for durable goods is needed in consumer research. We hope that the current research stimulates extent research on such factors.

We discuss some venues for future research.
We tested our hypotheses with respect to the preference for consumer electronics such as smartphones and washing machines. Among various types of durable goods consumers buy, they are relatively expensive and have long usage durations. Future research may test whether similar effects would emerge for durables that are less expensive and has shorter usage duration. Similarly, future research may examine whether there exists a future contraction effect for services. Just like durable goods, services also provide an extended stream of benefits. Then, consumers may contract service periods starting in the future. For instance, when consumers are deciding whether to pay for the gym membership fee today or do so from next month, they may perceive the duration of membership to be shorter when it starts in the future than immediately. We hope to address this question in the future research.

We acknowledge some limitations of the current research. First, we tested our hypotheses using hypothetical scenarios. Although hypothetical scenarios are commonly used in studying consumers' intertemporal choice decisions, consumers may behave differently when they make decisions for real outcomes. Second, all of our participants were residents of United States. Although we believe that the relationship between the future contraction effect and intertemporal preference for durable goods is a general phenomenon, consumers in different cultural background may be different in time perception and in their 
attitudes toward impatient consumptions. Studying our hypotheses among participants with different cultures will enlarge the generalizability of our findings. Third, to test our findings in different settings, the amount of rewards should be re-calibrated according to the market situation. For instance, in Study 2 we presented participants with a reward of $\$ 100$ for a smartphone delayed by 12 months. We used this amount based on our own market research for the smartphone market during the time we ran the study. Because the product cycle of smartphones gets shorter and the price of smartphones becomes higher these days, however, this amount would not be enough as compensation for a smartphone delayed by 12 months. Although we did not address these limitations in the current research to reduce the scope of our investigation, we plan to address them in our future studies.

\section{Conclusion}

Usage duration is the key defining property of durables. However, research on the subjective nature of usage duration perception and its role in consumer behavior is still very scant. We hope that our research stimulates various future studies regarding consumers' perception of usage duration as well as its impact on their preference and choice. These studies would enlighten our understanding of consumer behavior and contribute to the increase of consumer well-being.

〈Received March 27. 2018〉

〈Accepted July 20. 2018〉

\section{References}

Bartels, Daniel M. and Lance J. Rips (2010), "Psychological Connectedness and Intertemporal Choice," Journal of experimental psychology: General, 139, 49-69. and Oleg Urminsky (2011), “On

Intertemporal Selfishness: How the Perceived Instability of Identity Underlies Impatient Consumption," Journal of Consumer Research, 38, 182-98.

Bickel, Warren K. and Lisa A. Marsch (2001), "Toward a behavioral economic understanding of drug dependence: delay discounting processes," Addiction, 96(1), 73-86.

Bilgin, Baler and Robyn A. LeBoeuf (2010), "Looming Losses in Future Time Perception," Journal of Marketing Research, 47, 520-30. Chabris, Christopher F., David Laibson, Carrie L. Morris, Jonathon P. Schuldt and Dmitry Taubinsky (2008), "Individual laboratorymeasured discount rates predict field behavior," Journal of Risk and Uncertainty, $37(2-3), 237$.

Ebert, Jane E. and Drazen Prelec (2007), “The Fragility of Time: Time-Insensitivity and 
Valuation of the Near and Far Future," Management Science, 53, 1423-38.

Hardisty, David and Elke Weber (2009),

"Discounting Future Green: Money vs. the

Environment," Journal of Experimental

Psychology: General, 138, 329-40.

Killeen, Peter R. (2009), “An Additive-Utility Model of Delay Discounting," Psychological Review, 116, 602-19.

Kim, B. Kyu and Gal Zauberman (2009), "Perception of Anticipatory Time in Temporal Discounting," Journal of Neuroscience, Psychology, and Economics, 2, 91-101.

Kirby, Kris N. and Nancy M. Petry (2004), "Heroin and cocaine abusers have higher discount rates for delayed rewards than alcoholics or non-drug-using controls," Addiction, 99, 461-471.
Loewenstein, George (1996), "Out of control: Visceral influences on behavior," Organizational Behavior and Human Decision Processes, 65(3), 272-292. and Drazen Prelec (1992), "Anomalies in Intertemporal Choice: Evidence and an Interpretation," The Quarterly Journal of Economics, 107, 573-97. Stevens, Stanley S. (1957), "On the Psychophysical Law," Psychological Review, 64, 153-81. Thaler, Richard (1981), "Some empirical evidence on dynamic inconsistency," Economic Letters, 8(3), 201-207.

Zauberman, Gal, B. Kyu Kim, Selin Malkoc, James R. Bettman (2009), “Time Discounting and Discounting Time," Journal of Marketing Research, 46, 543-56. 